\title{
A Two-step Strategy for Stabilizing Control of Quantum Systems with Uncertainties *
}

\author{
Bo Qi ${ }^{\text {a,b }}$ \\ ${ }^{a}$ Key Laboratory of Systems and Control, ISS, Academy of Mathematics and Systems Science, Chinese Academy of Sciences, Beijing \\ 100190, P. R. China \\ ${ }^{\mathrm{b}}$ Centre for Quantum Computation and Communication Technology (Australian Research Council), Centre for Quantum Dynamics, \\ Griffith University, Brisbane, QLD 4111, Australia
}

\begin{abstract}
As a matter of course, designed control laws are to be acted on actual systems. However, most designed control laws are proven to be effective when performed on ideal models of the systems as opposed to the actual systems themselves. One fundamental problem is how to ensure the satisfactory performance of the designed control laws when they are performed on actual systems. Focusing on the state stabilization of quantum systems, a two-step strategy is proposed to solve this problem. A feedback strategy and the open-loop control technique of dynamical decoupling are combined therein to deal with the inevitable differences between an actual system and its model, by taking advantage of the distinct quantum characteristics: the measurement-induced-state-transfer and the tensor product structure, with the structure characterizing the coupling between a quantum system and its environment. Specifically, in the first step, a measurementbased feedback control strategy is selected according to a model of the actual system. In the second step, by identifying the differences between the actual system and the established model as decoherence noise, a specific control procedure is designed through dynamical decoupling. This procedure allows the realization of the chosen control strategy so as to deal with the differences.
\end{abstract}

Key words: State Stabilization; Measurement-based Feedback Control; Dynamical Decoupling; Two-step Strategy

\section{Introduction}

Due to potentially powerful capabilities of quantum information technology, quantum engineering has been a hot topic of intense research in recent years. The demand for an exquisite degree of control over a quantum system to complete an assigned task is a key ingredient in the quantum information technology, such as robustly preparing a family of fiducial states (Bolognani \& Ticozzi, 2010). With sustained effort to the manipulation of quantum systems, much progress has been made in both theory and experiments (Sayrin, Dotsenko; Mirrahimi, Dotsenko; Zhang \& James, 2011; Yang, Lim; Gross, Strobel; Biercuk, Uys; Cui, Xi; Kuang \& Cong, 2008; Bermudez, Jelezko; Zhang, Wang; Qi, 2012), ranging from quantum optics and quantum dots, to trapped ions and

\footnotetext{
* This work was supported by the National Center for Mathematics and Interdisciplinary Sciences, CAS, and by the National Natural Science Foundation of China under Grant No. 61004049 and No. 61134008 . The preliminary result has been partially presented at the 31th Chinese Control Conference, Hefei, China, July 25-27, 2012. Corresponding author B. Qi. Tel.: +861062651439. Fax +861062587343.

Email address: qibo@amss.ac.cn (Bo Qi).
}

superconductors.

However, it is worth pointing out that there is still a big gap between theories being performed on actual systems and those being designed based on their models. Since a system of interest is generally open, the dynamics of its environment will affect its evolution. However, the environment may be so large and complicated that we are unable to gain all of its precise information. Hence, one has to isolate the system from its environment or simplify its environment with proper approximations to get an applicable model. Even when all the information of the total system can be obtained, its dynamics may be too cumbersome to be analyzed. As a result, an appropriate simplified model also has to be set up for effective analysis. Hence, it is imperative to build a suitable model of the system for substantial research. In fact, hitherto most deliberately designed control laws and their corresponding satisfactory performance have been based on the systems' models. None the less, our intended concern is the effects of the control laws on the actual systems. Hence, more effort should be devoted to the fundamental question: how to ensure the satisfactory performance of the designed control laws when they are being performed on actual systems. 
The differences between the actual system and its built model can be regarded as some uncertainties, and there has been some research on dealing with these uncertainties in quantum control theory. For example, an open-loop control technique of dynamical decoupling (DD) has been employed in suppressing decoherence of non-Markovian open quantum systems (Khodjasteh, Lidar; Khodjasteh \& Viola, 2009a,b; $\mathrm{Ng}$, Lidar). In James (Nurdin), a coherent feedback control strategy was used to suppress the effect of noise. In Li \& Khaneja (2009), an ensemble control method was introduced to robustly control a continuum of dynamical systems with different values of parameters characterizing different system dynamics. An LQG method was adopted in Stockton (Geremia) to estimate an external magnetic field despite the ignorance of the size of the spin ensemble. A robust control approach based on sliding mode design was proposed in Dong \& Petersen (2012) for two-level systems with bounded uncertainties. By focusing on the state stabilization of quantum systems, this paper presents a two-step strategy to solve the identified problem. The advantages of feedback control and open-loop control are combined to deal with the differences between the actual systems and their models.

Feedback is an essential concept in control theory (Bolognani \& Ticozzi, 2010; Mirrahimi, Dotsenko; Zhang, Wu; Wiseman \& Milburn, 2010; Xie \& Guo, 2000), through which various methods for dealing with uncertainties can be developed. In the first step of our two-step strategy, adopting the idea of measurement-based feedback, we first set up a model based on the obtained information from the system, and then determine an appropriate map from the measurement outcomes to the ideal unitary transformations that should be applied. In the second step, considering the differences between the system and its model as some uncertainties which may lead to decoherence, we employ the powerful DD technique to realize the ideal unitary transformations designed in the first step with a high level of accuracy.

It is worth pointing out that in our two-step strategy, not only feedback but also the open-loop control technique is taken full advantage of in dealing with the differences between the quantum system and its model. It will be shown that the quantum characteristics, specifically, the measurementinduced-state-transfer and the tensor product structure characterizing the coupling between a quantum system and its environment, play important roles in the control process.

The paper is organized as follows. In Section 2, the control problem is formulated. In Section 3 and 4, we give the twostep strategy and analyze its effect, respectively. Section 5 concludes the paper.

\section{The Control Problem}

Let $S$ denote the system of interest, coupled to its environment $E$, with respective Hilbert space $\mathscr{H}_{S}$ and $\mathscr{H}_{E}$. We assume the Hamiltonian of the total system can be described as

$$
H_{t o t}=H_{S, 0}+H_{S, e}+H_{E}+H_{S E}+H_{C},
$$

where $H_{S, 0}$ is the known Hamiltonian of system $S$, while $H_{S, e}$ accounts for the part of system $S$ that we cannot identify exactly. $H_{E}$ is the Hamiltonian of the environment, and $H_{S E}$ describes the coupling between $S$ and $E$. Suppose we only know the structures of the Hamiltonian $H_{S, e}, H_{E}$, and $H_{S E}$, and they can be together described in the following form:

$$
H_{\Delta}=H_{S, e}+H_{E}+H_{S E}=\sum_{\alpha=1}^{N^{2}} S_{\alpha} \otimes E_{\alpha}
$$

where $N$ is the dimension of the Hilbert space $\mathscr{H}_{S} \cdot\left\{S_{\alpha}\right\}_{\alpha=1}^{N^{2}}$ is a complete basis of orthonormal operators of the corresponding Liouville space, namely $\operatorname{Tr}_{S}\left(S_{i}^{\dagger} S_{j}\right)=\delta_{i j}$, where $\dagger$ denotes the Hermitian adjoint, $\delta_{i j}$ is the Kronecker function. $E_{\alpha}, \alpha=1,2, \cdots, N^{2}$, are unknown but bounded operators acting on $\mathscr{H}_{E}$. For convenience, one of the basis operators is chosen to be proportional to the identity $I_{S}$, namely $S_{N^{2}}=(1 / N)^{\frac{1}{2}} I_{S}$, such that the other basis operators are traceless, that is $\operatorname{Tr}_{S}\left(S_{\alpha}\right)=0$, for $\alpha=1,2, \cdots, N^{2}-1$.

$H_{C}$ is the control Hamiltonian which we can adjust. Since our goal is to give a conceptual two-step control strategy for quantum systems, we assume that there is no imperfection in the controller part ${ }^{1}$, and that a universal control over $S$ is achievable (Nielsen \& Chuang, 2000).

Furthermore, suppose a set of generalized measurement operations $\left\{M_{k}\right\}_{k=1}^{K}\left(\sum_{k=1}^{K} M_{k}^{\dagger} M_{k}=I_{S}\right)$ can be performed on $S$, where $K$ is the maximum number of the possible measurement outcomes. In the following, we assume that the measurement time is much shorter than all other relevant time scales. Hence, the effect of $H_{\Delta}$ during the measurement process can be ignored. This generalized measurement and the Hamiltonian $H_{C}$ are all control means during the manipulation process.

Our aim is to asymptotically engineer quantum states of $S$ to a desired subset. Specifically, given a target subspace $\mathscr{H}_{I}$ of $\mathscr{H}_{S}$, the corresponding target subset $\mathscr{J}_{S}\left(\mathscr{H}_{I}\right)$ consists of states in the form of $\left(\begin{array}{cc}\rho_{I} & 0 \\ 0 & 0\end{array}\right)$, where $\rho_{I}$ is some state associated with the subspace $\mathscr{H}_{I}$. Note that if the dimension of the subspace $\mathscr{H}_{I}$ is 1 , the problem is equivalent to robustly preparing a desired pure state.

According to the target subspace $\mathscr{H}_{I}$, we can make a direct sum decomposition $\mathscr{H}_{S}=\mathscr{H}_{I} \oplus \mathscr{H}_{R}$, where $\mathscr{H}_{R}$ is the remainder of $\mathscr{H}_{I}$. This decomposition can induce a block structure for the matrix $X$ representing an operator acting

\footnotetext{
1 This kind of imperfection can be suppressed to an arbitrarily desired level by a classical control method.
} 
on $\mathscr{H}_{S}: X=\left(\begin{array}{cc}X_{I} & X_{P} \\ X_{Q} & X_{R}\end{array}\right)$, where $X_{I}, X_{P}, X_{Q}$, and $X_{R}$ are corresponding block matrices of $X$. In the rest of the paper the subscripts $I, P, Q$, and $R$ will follow this convention.

\section{The Two-step Control Strategy}

This section presents the proposed two-step strategy as follows.

Step 1. Set up an appropriate model and decide which unitary operation should be applied after each measurement.

First, isolate system $S$ from its environment according to the precise information that is obtained, i.e., the Hamiltonian of the model is:

$$
H_{\text {mod }}=H_{S, 0}+H_{C}
$$

Suppose the model state of system $S$ at step $t$ is $\hat{\rho}_{t}$. After the measurement, the $k$-th result will be obtained with probability $\operatorname{Tr}\left(M_{k}^{\dagger} M_{k} \hat{\rho}_{t}\right)$, and the corresponding quantum state will collapse to $\hat{\rho}_{t}^{k}=\frac{M_{k} \hat{\rho}_{t} M_{k}^{\dagger}}{\operatorname{Tr}\left(M_{k}^{\dagger} M_{k} \hat{\rho}_{t}\right)}$. Then one should design a map from the set of measurement outcomes to the set of unitary operations $\left\{U_{k}\right\}_{k=1}^{K}$, which are to be acted on $S$ in order to control the evolution of state $\hat{\rho}_{t}$. Should the $k$-th outcome appear, $U_{k}$ is to be applied to the model for it to evolve to $U_{k} \hat{\rho}_{t}^{k} U_{k}^{\dagger}$.

It is well known that quantum mechanics only provides statistical properties of observables. Hence, only the average evolution of the quantum state is considered. At each step the measurement results are averaged, and this yields the evolution of the model state immediately after applying the unitary operations:

$$
\hat{\rho}_{t+1}=\varepsilon_{\{M, U\}}\left(\hat{\rho}_{t}\right)=\sum_{k} U_{k} M_{k} \hat{\rho}_{t} M_{k}^{\dagger} U_{k}^{\dagger},
$$

where the map $\varepsilon_{\{M, U\}}$ is a quantum operation.

Now, the subproblem that is presented is how to determine a map from the set of the measurement operators $\left\{M_{k}\right\}_{k=1}^{K}$ to the set of $\left\{U_{k}\right\}_{k=1}^{K}$, to asymptotically engineer the state described by eq. (3) to the desired set $\mathscr{J}_{S}\left(\mathscr{H}_{I}\right)$.

First, we recall three definitions (Bolognani \& Ticozzi, 2010). Let $S$ evolve under iterations of the quantum operation $\mathscr{T}$ :

$$
\mathscr{T}(\rho)=\sum_{k} M_{k} \rho M_{k}^{\dagger}
$$

where $\rho$ is a density operator.

Definition 1 (Invariance). The set $\mathscr{J}_{S}\left(\mathscr{H}_{I}\right)$ is invariant if the evolution of any initialized $\rho_{0} \in \mathscr{J}_{S}\left(\mathscr{H}_{I}\right)$ obeys $\rho_{t}=\mathscr{T}^{t}\left(\rho_{0}\right)$ $\in \mathscr{J}_{S}\left(\mathscr{H}_{I}\right)$ for any $t \geq 0$, where $\mathscr{T}^{t}$ indicates $t$ applications of the map $\mathscr{T}$.

Definition 2 (Attractivity). The set $\mathscr{J}_{S}\left(\mathscr{H}_{I}\right)$ is attractive if for arbitrary $\rho$ of $S$, we have

$$
\lim _{t \rightarrow \infty}\left\|\mathscr{T}^{t}(\rho)-\Pi_{I} \mathscr{T}^{t}(\rho) \Pi_{I}\right\|=0,
$$

where $\Pi_{I}$ is the projection operator over the subspace $\mathscr{H}_{I}$, and $\|A\|=\sqrt{\operatorname{Tr}\left(A^{\dagger} A\right)}$.

Definition 3 (Global Asymptotic Stability). The set $\mathscr{J}_{S}\left(\mathscr{H}_{I}\right)$ is globally asymptotically stable (GAS) if it is invariant and attractive.

To solve the identified subproblem, we can employ the constructive algorithm in Theorem 5 in Bolognani \& Ticozzi (2010), whose basic idea is the following theorem:

Theorem 1 ( Theorem 2 in Bolognani \& Ticozzi (2010)) Let the quantum operation $\mathscr{T}$ be described by the Kraus map $\mathscr{T}(\rho)=\sum_{k} M_{k} \rho M_{k}^{\dagger}$. Consider an direct sum decomposition $\mathscr{H}_{S}=\mathscr{H}_{I} \bigoplus \mathscr{H}_{R}$, with $\mathscr{J}_{S}\left(\mathscr{H}_{I}\right)$ invariant. Let the matrix $M_{k}$ be expressed in its block form $M_{k}=\left(\begin{array}{cc}M_{I, k} & M_{P, k} \\ M_{Q, k} & M_{R, k}\end{array}\right)$, according to the same state space decomposition. Then the set $\mathscr{J}_{S}\left(\mathscr{H}_{I}\right)$ is GAS if and only if there are no invariant states with support on $\bigcap_{k} \operatorname{ker}\left(M_{P, k}\right)$, where $\operatorname{ker}(X)$ is the kernel (O-eigenspace) of $X$.

By an algebraic characterization of the measurement operators $\left\{M_{k}\right\}_{k=1}^{K}$, Theorem 5 in Bolognani \& Ticozzi (2010) clearly showed for a given set of $\left\{M_{k}\right\}_{k=1}^{K}$, whether or not a set of unitary operations $\left\{U_{k}\right\}_{k=1}^{K}$ can be found to make that there are no invariant states with support on $\bigcap_{k} \operatorname{ker}\left(N_{P, k}\right)$, where $N_{k}=U_{k} M_{k}$. If this is possible, then from (3) and Theorem 1 , we can conclude that the desired subset $\mathscr{J}_{S}\left(\mathscr{H}_{I}\right)$ is asymptotically stable for the model dynamics (3). Moreover, in this case Theorem 5 in Bolognani \& Ticozzi (2010) provided an iterative control design procedure that can return an effective control choice $\left\{U_{k}\right\}_{k=1}^{K}$ in $N$ steps at the most .

In Section 4, we will explicitly give the condition of $\left\{M_{k}\right\}_{k=1}^{K}$ that can make us find a set of $\left\{U_{k}\right\}_{k=1}^{K}$ to complete the control task with the model system, while omit the detailed procedure to get the control operations $\left\{U_{k}\right\}_{k=1}^{K}$ which is the same as in Theorem 5 in Bolognani \& Ticozzi (2010).

Remark 1 For a real system, the undertaking of measurement requires time. Usually the measurement operator $M_{k}$ does not commute with the Hamiltonian of the system. In this paper, for simplicity, we assume that the measurement time scale is much shorter than all other time scales. Under this assumption, we can apply the measurement and unitary transformation on the system sequentially as in eq. (3). Moreover, since $\sum_{k} M_{k}^{\dagger} U_{k}^{\dagger} U_{k} M_{k}=I$, we may consider 
$\left\{U_{k} M_{k}\right\}_{k=1}^{K}$ as a new set of generalized measurement operators. Note that in Step 1, it is the measurement-inducedstate-transfer effect that is employed to asymptotically engineer the model state to the target set $\mathscr{J}_{S}\left(\mathscr{H}_{I}\right)$.

Step 2. By considering the differences between the quantum system and its model as some decoherence noise, a specific control procedure can be designed through employing the powerful DD technique to realize the unitary operators $\left\{U_{k}\right\}_{k=1}^{K}$ with high fidelity so as to deal with the differences.

Step 1 shows that given a proper set of measurement operators $\left\{M_{k}\right\}_{k=1}^{K}$, one can design a set of unitary transformations $\left\{U_{k}\right\}_{k=1}^{K}$ to complete the control task with the ideal model (corresponding to the case of $H_{\Delta}=H_{S, e}+H_{E}+H_{S E}=0$ ). However, for an actual system, the Hamiltonian $H_{\Delta}$ is generally not zero. Hence, the system $S$ and its environment $E$ may be coupled together, and one has to trace out the environment in order to get the state $\rho_{t}$ describing the actual dynamics of system $S$. In this sense, $H_{\Delta}$ can be treated as decoherence noise.

According to the measurement-control process proposed in Step 1, the dynamics of $\rho_{t}$ is:

$$
\begin{aligned}
\rho_{t+1} & =\varepsilon\left(\rho_{t}\right) \\
& =\sum_{k} \operatorname{Tr}\left(M_{k}^{\dagger} M_{k} \rho_{t}\right) \operatorname{Tr}_{E}\left\{U_{k, t o t}\left(\rho_{t}^{k} \otimes \rho_{t, E}^{k}\right) U_{k, t o t}^{\dagger}\right\} \\
& =\sum_{k} \operatorname{Tr}_{E}\left\{U_{k, t o t}\left(M_{k} \rho_{t} M_{k}^{\dagger} \otimes \rho_{t, E}^{k}\right) U_{k, t o t}^{\dagger}\right\},
\end{aligned}
$$

where the map $\varepsilon$ is a quantum operation, $\rho_{t}^{k}=\frac{M_{k} \rho_{t} M_{k}^{\dagger}}{\operatorname{Tr}\left(M_{k}^{\dagger} M_{k} \rho_{t}\right)}$ is the state of the system after the $k$-th outcome being obtained, while $\rho_{t, E}^{k}$ is the corresponding state of the environment at that time. $U_{k, t o t}$ is the actual unitary evolution of the total system when $U_{k}$ is expected to be applied on system $S$.

It can be clearly seen that the actual evolution in eq. (4) is different from the ideal dynamics in eq. (3), owing to the nonzero error Hamiltonian $H_{\Delta}$. Although the precise information of $H_{\Delta}$ is not obtained, its algebraic form is known, i.e., eq. (2). Now $H_{\Delta}$ is regarded as a term which may lead to decoherence, and the DD technique is employed to suppress its effect so as to realize the ideal $\left\{U_{k}\right\}_{k=1}^{K}$ with a high level of accuracy.

Suppose the initial state is the same $\rho_{S}^{0}$ for the model and the actual system. The model state after the unitary transformation $U$ is $\rho_{U} \doteq U \rho_{S}^{0} U^{\dagger}$, while the corresponding actual system state after the total unitary evolution $U_{t o t}$ is

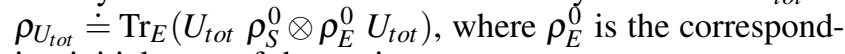
ing initial state of the environment.

Now, we define the trace distance between $\rho_{U}$ and $\rho_{U_{t o t}}$ as

$$
D\left(\rho_{U}, \rho_{U_{t o t}}\right)=\frac{1}{2} \operatorname{Tr}\left|\rho_{U}-\rho_{U_{t o t}}\right|,
$$

where $|A|=\sqrt{A^{\dagger} A}$. The distance $D\left(\rho_{U}, \rho_{U_{t o t}}\right)$ is used to characterize how the ideal unitary transformation $U$ can be realized by $U_{t o t}$. Note that the smaller the trace distance is, the more accurately $U$ can be realized.

Our goal is to synthesize a control modulation which can approximate $U$ with an error scaling as $O\left(\tau_{0}^{l+1}\right)$, where $\tau_{0}$ is the minimum duration over which the control Hamiltonian $H_{C}(t)$ is affected, and $l$ is a positive integer. The synthesized total unitary transformation is denoted by $U_{t o t}^{[l]}$ with total time duration denoted by $\tau_{l}$.

In Khodjasteh (Lidar), a constructive algorithm is given to synthesize unitary transformations with an arbitrarily high level of accuracy at the price of nested structures and long time duration. The main idea is DD, which is a powerful and versatile technique for suppressing decoherence in nonMarkovian open quantum systems.

Now suppose that there exists a decoupling group $\mathscr{G}=\left\{G_{i}\right\}$ for $\left\{S_{\alpha}\right\}_{\alpha=1}^{N^{2}-1}$, with order $|\mathscr{G}|=d$ and $m$ group generators $F=\left\{F_{j}\right\}_{j=1}^{m}$, i.e.,

$$
\sum_{i=1}^{d} G_{i}^{\dagger} S_{\alpha} G_{i}=0, \quad \text { for } \alpha=1,2, \cdots, N^{2}-1
$$

From Khodjasteh (Lidar), for any ideal unitary transformation $U$ and positive integer $l$, a control modulation can be recursively constructed such that the net error $D\left(\rho_{U}, \rho_{U_{\text {tot }}^{[l]}}\right)$ is bounded by a function of $\tau_{0}$, denoted as $\Delta\left(\tau_{0}\right)$ with the definition as

$$
\Delta\left(\tau_{0}\right) \doteq\left(\chi_{l}\right)^{l^{2}} c\left\|H_{S E}+H_{S, e}\right\|\left(4 \chi_{l} \tau_{0}\left\|H_{\Delta}\right\|\right)^{l} \tau_{0},
$$

where $\chi_{l}=d(m+3)$, and $c=O(1)$. In order to get this order of the net error, the corresponding time duration $\tau_{l}$ satisfies

$$
\tau_{l}=\left[d m+(d-1)\left(1+2^{\frac{1}{l}}\right)+3\right] \tau_{l-1}, \quad l \geq 1 .
$$

From (6), we can see that if the control modulation is fast enough, i.e., $\tau_{0}$ is sufficiently small, then the upper bound of the net error $\Delta\left(\tau_{0}\right)$ can be arbitrarily small. Hence, an ideal unitary transformation can be realized with an arbitrarily high level of accuracy.

Remark 2 From (2) and (5), one can see that the distinc$t$ property of the quantum system, i.e., the tensor product structure between $S$ and its environment $E$, is tactically utilized by the open-loop control technique of DD to suppress the effect of the error Hamiltonian $H_{\Delta}$. Hence, the openloop control technique has been used in the second step to deal with the differences between the quantum system and its model. This is significantly different from the control of classical systems. 
Remark 3 Note that in step 2, although we use a specific powerful DD technique to realize the chosen unitary transformations with high fidelity, the total frame is different. Our aim is to combine the advantages of the feedback control and open-loop control to provide a possible solution to the identified problem. It is worth pointing out that the extensive time is required to obtain a high level of accuracy when synthesizing an ideal unitary transformation using the DD technique, the total duration is very long. Moreover, DD is effective only against low-frequency noise, and an enough rate of control modulation should be employed. Further research will involve the exploration of other efficient methods to complete the identified task.

\section{Performance Evaluation}

In this section, we combine the results of Step 1 and Step 2 to analyze the effect of the two-step strategy in achieving our control objective.

We first define the distance between a state $\rho$ and the target set $\mathscr{J}_{S}\left(\mathscr{H}_{I}\right)$ as

$$
D\left(\rho, \mathscr{J}_{S}\left(\mathscr{H}_{I}\right)\right)=\inf _{\sigma \in \mathscr{J}_{S}\left(\mathscr{H}_{I}\right)} D(\rho, \sigma) .
$$

Consider the canonical QR decomposition of the measurement operator $M_{k}=Q_{k} R_{k}$ (Bolognani \& Ticozzi, 2010), where $Q_{k}$ is an orthogonal matrix (meaning that $Q_{k}^{\dagger} Q_{k}=I$ ) and $R_{k}$ is an upper triangular matrix. Recall that according to the direct sum decomposition $\mathscr{H}_{S}=\mathscr{H}_{I} \bigoplus \mathscr{H}_{R}$, where $\mathscr{H}_{I}$ is the desired subspace, and $\mathscr{H}_{R}$ is its remainder, the corresponding block structure of a matrix $X$ acting on $\mathscr{H}_{S}$ is $X=\left(\begin{array}{ll}X_{I} & X_{P} \\ X_{Q} & X_{R}\end{array}\right)$. Let $R_{k}$ be expressed in its block form

$$
R_{k}=\left(\begin{array}{cc}
R_{I, k} & R_{P, k} \\
R_{Q, k} & R_{R, k}
\end{array}\right)
$$

Now we can give the main theorem:

Theorem 2 Under the two-step strategy as described in Section 3 , the distance $D\left(\rho_{t}, \mathscr{J}_{S}\left(\mathscr{H}_{I}\right)\right)$ between the system state $\rho_{t}$ and the target subset $\mathscr{J}_{S}\left(\mathscr{H}_{I}\right)$ can be arbitrarily small in finite steps, if the following conditions are satisfied:

A1. For the canonical $Q R$ decomposition of the measurement operators $M_{k}=Q_{k} R_{k}$, there exists at least one $R_{P, k} \neq$ 0 , and $\bigcap_{k} \operatorname{ker}\left(R_{P, k}\right)=\{0\}$

A2. A universal control over $S$ is achievable through control Hamiltonian $H_{C}$, and there exists a decoupling group $\mathscr{G}$ for $\left\{S_{\alpha}\right\}$, i.e., eq. (5) is met;

A3. $H_{\Delta}$ is bounded, and the rate of the control modulation is fast enough, i.e., $\tau_{0}$ is sufficiently small.
Proof. It is clear that

$$
D\left(\rho_{t}, \mathscr{J}_{S}\left(\mathscr{H}_{I}\right)\right) \leq D\left(\hat{\rho}_{t}, \mathscr{J}_{S}\left(\mathscr{H}_{I}\right)\right)+D\left(\rho_{t}, \hat{\rho}_{t}\right)
$$

where $\rho_{t}\left(\hat{\rho}_{t}\right)$ is the actual (model) state of system $S$, evolving as eq. (4) (eq. (3)). Hence, we just need to estimate $D\left(\hat{\rho}_{t}, \mathscr{J}_{S}\left(\mathscr{H}_{I}\right)\right)$ and $D\left(\rho_{t}, \hat{\rho}_{t}\right)$.

In order to estimate $D\left(\hat{\rho}_{t}, \mathscr{J}_{S}\left(\mathscr{H}_{I}\right)\right)$, from Theorem 5 in Bolognani \& Ticozzi (2010), we conclude that if at least one $R_{P, k} \neq 0$ and $\bigcap_{k} \operatorname{ker}\left(R_{P, k}\right)=\{0\}$, one can find a set of unitary operators $\left\{U_{k}\right\}$ such that $\hat{\rho}_{t}$ can be engineered to the desired set $\mathscr{J}_{S}\left(\mathscr{H}_{I}\right)$ asymptotically. The dynamics of $\hat{\rho}_{t}$ can be further described as

$$
\hat{\rho}_{t+1}=\sum_{k} N_{k} \hat{\rho}_{t} N_{k}^{\dagger}
$$

where $N_{k}=U_{k} M_{k}$, satisfying $\sum_{k} N_{k}^{\dagger} N_{k}=I$ and $\bigcap_{k} \operatorname{ker}\left(N_{P, k}\right)=$ $\{0\}$.

Note that if $\bigcap_{k} \operatorname{ker}\left(N_{P, k}\right)=\{0\}$, it is not difficult to get

$$
\sum_{k} N_{P, k}^{\dagger} N_{P, k}>0
$$

From $\sum_{k} N_{k}^{\dagger} N_{k}=I$ and $\sum_{k} N_{P, k}^{\dagger} N_{P, k}>0$, it is clear that

$$
\sum_{k} N_{R, k}^{\dagger} N_{R, k}<I
$$

From (8), one has

$$
\hat{\rho}_{t+1, R}=\sum_{k} N_{R, k} \hat{\rho}_{t, R} N_{R, k}^{\dagger}
$$

Hence,

$$
\lambda\left(\hat{\rho}_{t+1, R}\right) \leq \operatorname{Tr}\left(\hat{\rho}_{t+1, R}\right) \leq \lambda\left(\sum_{k} N_{R, k}^{\dagger} N_{R, k}\right) \operatorname{Tr}\left(\hat{\rho}_{t, R}\right),
$$

where $\lambda(A)$ denotes the maximum eigenvalue of $A$. Hence, it is not difficult to see that

$$
\lambda\left(\hat{\rho}_{t+1, R}\right)=O\left(\lambda^{t}\left(\sum_{k} N_{R, k}^{\dagger} N_{R, k}\right)\right) .
$$

From the above equation and the definition of $D\left(\rho, \mathscr{J}_{S}\left(\mathscr{H}_{I}\right)\right)$, one gets

$$
D\left(\hat{\rho}_{t+1}, \mathscr{J}_{S}\left(\mathscr{H}_{I}\right)\right)=O\left(\lambda^{t}\left(\sum_{k} N_{R, k}^{\dagger} N_{R, k}\right)\right) .
$$

Since we have proved

$$
\sum_{k} N_{R, k}^{\dagger} N_{R, k}<I
$$


we get $\lambda\left(\sum_{k} N_{R, k}^{\dagger} N_{R, k}\right)<1$. Note that $\lambda^{t}\left(\sum_{k} N_{R, k}^{\dagger} N_{R, k}\right)$ is an exponential function with base $\lambda\left(\sum_{k} N_{R, k}^{\dagger} N_{R, k}\right)$ and variable $t$, therefore, $\hat{\rho}_{t}$ will converge exponentially to the desired set $\mathscr{J}_{S}\left(\mathscr{H}_{I}\right)$.

Now we estimate $D\left(\rho_{t}, \hat{\rho}_{t}\right)$. First we construct a reference process $\rho_{t}^{\prime}$ as

$$
\rho_{t+1}^{\prime}=\sum_{k} U_{k} M_{k} \rho_{t} M_{k}^{\dagger} U_{k}^{\dagger}
$$

where $\rho_{t}$ evolves as (4). From the triangle inequality of trace distance, one gets

$$
D\left(\rho_{t+1}, \hat{\rho}_{t+1}\right) \leq D\left(\rho_{t+1}^{\prime}, \hat{\rho}_{t+1}\right)+D\left(\rho_{t+1}, \rho_{t+1}^{\prime}\right) .
$$

Note that on one hand, from the property of quantum operations (Nielsen \& Chuang, 2000), one gets

$$
\begin{aligned}
D\left(\rho_{t+1}^{\prime}, \hat{\rho}_{t+1}\right) & =D\left(\varepsilon_{M, U}\left(\rho_{t}\right), \varepsilon_{M, U}\left(\hat{\rho}_{t}\right)\right) \\
& \leq D\left(\rho_{t}, \hat{\rho}_{t}\right)
\end{aligned}
$$

On the other hand, from the property of trace distance (Nielsen \& Chuang, 2000), one has

$$
\begin{aligned}
& D\left(\rho_{t+1}, \rho_{t+1}^{\prime}\right) \\
= & D\left(\sum_{k} \operatorname{Tr}\left(M_{k}^{\dagger} M_{k} \rho_{t}\right) \operatorname{Tr}_{E}\left\{U_{k, t o t}\left(\rho_{t}^{k} \otimes \rho_{t, E}^{k}\right) U_{k, t o t}^{\dagger}\right\}\right. \\
& \left.\sum_{k} \operatorname{Tr}\left(M_{k}^{\dagger} M_{k} \rho_{t}\right) U_{k} \rho_{t}^{k} U_{k}^{\dagger}\right) \\
\leq & \sum_{k} \operatorname{Tr}\left(M_{k}^{\dagger} M_{k} \rho_{t}\right) D\left(\operatorname{Tr}_{E}\left\{U_{k, t o t}\left(\rho_{t}^{k} \otimes \rho_{t, E}^{k}\right) U_{k, t o t}^{\dagger}\right\}, U_{k} \rho_{t}^{k} U_{k}^{\dagger}\right)
\end{aligned}
$$

From inequalities (6), (11)-(13), condition A2, and $\rho_{0}=\hat{\rho}_{0}$, it is clear that

$$
D\left(\rho_{t+1}, \hat{\rho}_{t+1}\right)=(t+1) O\left(\Delta\left(\tau_{0}\right)\right)
$$

Combining inequalities (6), (7), (9), and (15), we have

$$
D\left(\rho_{t}, \mathscr{J}_{S}\left(\mathscr{H}_{I}\right)\right)=O\left(\lambda^{t-1}\left(\sum_{k} N_{R, k}^{\dagger} N_{R, k}\right)\right)+t O\left(\Delta\left(\tau_{0}\right)\right) .
$$

Hence, we conclude from condition A3 that the control objective can be achieved satisfactorily after finite steps.

Remark 4 In the measurement-based feedback control of quantum systems, measurement itself can be considered as a control means due to the measurement-induced-statetransfer effect. Hence, one has to choose the measurement operators appropriately in order to achieve the quantum control target. Condition Al is actually a technical assumption. It may be a little limited, but it does give a criterion of how to choose the measurement operators. If there exists at least one $R_{P, k} \neq 0$, then unitary operations $\left\{U_{k}\right\}_{k=1}^{K}$ can be found to make the desired subset asymptotically stable for the model dynamics. $\bigcap_{k} \operatorname{ker}\left(R_{P, k}\right)=\{0\}$ further guarantees that the convergence rate of the model state to the desired subset is exponential.

Remark 5 Conditions $A 2$ and A3 may be realized in spin systems. For example, the system $S$ may be an electron spin, while its environment may consist of a finite number of nuclear spins. For a spin system, it is easy to see that the decoupling group can be the identity and Pauli matrices, i.e., $\mathscr{G}=\left\{I, X=\left(\begin{array}{ll}0 & 1 \\ 1 & 0\end{array}\right), Y=\left(\begin{array}{cc}0 & -i \\ i & 0\end{array}\right), Z=\left(\begin{array}{cc}1 & 0 \\ 0 & -1\end{array}\right)\right\}$. The error Hamiltonian $H_{\Delta}$ is bounded owing to the limited environment. Furthermore, thanks to the rapid development of the ultra fast pulse technique, the rate of control modulation can be made sufficiently fast.

Remark 6 From (3), (10) and (12), one can see that the measurement-induced-state-transfer effect plays a positive role in dealing with the differences between the actual and ideal evolution of the state.

\section{Conclusion}

In this paper, we combine the concepts of feedback and the open-loop control technique to present a two-step strategy by focusing on a control problem of quantum state stabilization. This two-step strategy is used to investigate the fundamental question: how to ensure the satisfactory performance of the control laws when they are performed on actual systems. In the proposed strategy, we first set up a model based on the available information, and decide the corresponding control strategy. We then employ the DD technique to realize the design strategy to deal with the inevitable differences between the system and the established model. It is expected that the proposed approach will trigger more studies aiming to bridge control theories being designed based on the models as opposed to actual systems.

\section{Acknowledgement}

The author thanks L. Guo and H. M. Wiseman for helpful discussion.

\section{References}

Bermudez, A., Jelezko, F., Plenio, M. B., \& Retzker, A. (2011). Electron-mediated nuclear-spin interactions between distant nitrogen-vacancy centers. Physical Review Letters, 107, 150503.

Biercuk, M. J., Uys, H., VanDevender, A. P., Shiga, N., Itano, W. M., \& Bollinger, J. J. (2009). Optimized dynamical decoupling in a model quantum memory, Nature, 458, 996-1000. 
Bolognani, S., \& Ticozzi, F. (2010). Engineering stable discrete-time quantum dynamics via a canonical QR decomposition. IEEE Transactions on Automatic Control, $55,2721-2734$.

Cui, W., Xi, Z. R., \& Pan, Y. (2010). Non-Markovian entanglement dynamics in coupled superconducting qubit systems. The European Physical Journal D, 59, 479-485.

Dong, D., \& Petersen, I. R. (2012). Sliding mode control of two-Level quantum systems. Automatica, 48, 725-735.

Gross, C., Strobel, H., Nicklas, E., Zibold, T., Bar-Gill, N., Kurizki, G., \& Oberthaler M. K. (2011). Atomic homodyne detection of continuous-variable entangled twinatom states. Nature, 480, 219-223.

James, M. R., Nurdin, H. I., \& Petersen, I. R. (2008). $H^{\infty}$ control of linear quantum systems. IEEE Transactions on Automatic Control, 53, 1787-1803.

Khodjasteh, K., Lidar, D. A., \& Viola, L. (2010). Arbitrarily accurate dynamical control in open quantum systems. Physical Review Letters, 104, 090501.

Khodjasteh, K., \& Viola, L. (2009a). Dynamically errorcorrected gates for universal quantum computation. Physical Review Letters, 102, 080501.

Khodjasteh, K., \& Viola, L. (2009b). Dynamical quantum error correction of unitary operations with bounded controls. Physical Review A, 80, 032314.

Kuang, S., \& Cong, S. (2008). Lyapunov control methods of colsed quantum systems. Automatica, 44, 98-108.

Li, J. S., \& Khaneja, N. (2009). Ensemble control of bloch equations. IEEE Transactions on Automatic Control, 54, 528-536.

Mirrahimi, M., Dotsenko, I., \& Rouchon P. (2009). Feedback generation of quantum Fock states by discrete QND measures. In Joint 48th IEEE Conference on Decision and Control and 28th Chinese Control Conference, pp.14511456, Shanghai, P. R. China, December.

Ng, H. K., Lidar, D. A., \& Preskill, J. (2011). Combining dynamical decoupling with fault-tolerant quantum computation. Physical Review A, 84, 012305.

Nielsen, M., \& Chuang, I. (2000). Quantum computation and quantum information. Cambridge: Cambridge University Press.

Qi, B. (2012). Stabilizing control of quantum Systems with uncertainties. Proceedings of the 31st Chinese Control Conference. pp.25-27, Hefei, P. R. China, July.

Sayrin, C., Dotsenko, I., Zhou, X. X., Peaudederf, B., Rybarczyk, T., Gleyzes, S., Rouchon, P., Mirrahimi, M., Amini, H., Brune, M., Raimond, J.-M., \& Haroche S. (2011). Real-time quantum feedback prepares and stabilizes photon number states. Nature, 477, 73-77.

Stockton, J. K., Geremia, J. M., Doherty, A. C., \& Mabuchi, H. (2004). Robust quantum parameter estimation: Coherent magnetometry with feedback. Physical Review A, 69, 032109.

Wiseman, H. M. \& Milburn, G. J. (2010). Quantum Measurement and Control. Cambridge: Cambridge University Press.

Xie, L. L., \& Guo, L. (2000). How much uncertainty can be dealt with by feedback? IEEE Transactions on Automatic Control, 45, 2203-2217.
Yang, C. H., Lim, W. H., Zwanenburg, F. A., \& Dzurak, A. S. (2011). Dynamically controlled charge sensing of a fewelectron silicon quantum dot. AIP Advances, 1, 042111.

Zhang, G. F. \& James, M. R. (2011). Direct and indirect couplings in coherent feedback control of linear quantum systems. IEEE Transactions on Automatic Control, 56, 1535-1550.

Zhang, J., Wu, R. B., Li, C. W., \& Tarn, T. J. (2010). Protecting coherence and entanglement by quantum feedback controls, IEEE Transactions on Automatic Control, 55, 619-633.

Zhang, M., Wang, W. G., Dai, H. Y., \& Xi, Z. R. (2009). Evolution of two non-identical two-level atoms in two-mode cavity fields. Communication in Theoretical Physics, 51, 215-220. 